\title{
Probing Proteostatic Stress in Degenerating Photoreceptors Using Two Complementary In Vivo Reporters of Proteasomal Activity
}

\author{
Paige M. Dexter, ${ }^{1}$ Ekaterina S. Lobanova, ${ }^{2}$ Stella Finkelstein, ${ }^{2}$ and ${ }^{\circledR}$ Vadim Y. Arshavsky ${ }^{1,2}$
}

https://doi.org/10.1523/ENEURO.0428-19.2019

${ }^{1}$ The Department of Pharmacology and Cancer Biology, Duke University School of Medicine, Durham, NC 27710 and

${ }^{2}$ Albert Eye Research Institute, Duke University, Durham, NC 27710

\begin{abstract}
Inherited retinal degenerations originate from mutations in $>300$ genes, many of which cause the production of misfolded mutant photoreceptor proteins that are ultimately degraded by the ubiquitin-proteasome system (UPS). It was previously shown that rod photoreceptors in multiple mouse models of retinal degeneration suffer from proteostatic stress consisting of an insufficient cellular capacity for degrading UPS substrates. In this study, we focused on a specific UPS component required for the degradation of a subset of proteasome targets: the substrate-processing complex formed by the AAA + ATPase P97/NCP and associated cofactors. To assess whether P97 capacity may be insufficient in degenerating rods, we employed two complementary in vivo proteasomal activity reporters whose degradation is either P97-dependent or P97-independent. Retinal accumulation of each reporter was measured in two models of retinal degeneration: the transducin $\gamma$-subunit knock-out $\left(\mathrm{G} \gamma_{1}{ }^{-/}\right)$and $\mathrm{P} 23 \mathrm{H}$ rhodopsin knock-in (P23H) mice. Strikingly, the patterns of reporter accumulation differed between these models, indicating that the proteostatic stress observed in $\mathrm{G} \gamma_{1}^{-/-}$and $\mathrm{P} 23 \mathrm{H}$ rods likely originates from different pathobiological mechanisms, in which UPS substrate degradation may or may not be limited by P97-dependent substrate processing. Further, we assessed whether P97 overexpression could ameliorate pathology in $\mathrm{G} \gamma_{1}^{-/-}$mice, in which proteostatic stress appears to result from P97 insufficiency. However, despite P97 overexpression being aphenotypic in other tissues, the $\sim 2$.4-fold increase in retinal P97 content was toxic to rods, which complicated the interpretation of the observed phenotype. Our results highlight the complexity of pathophysiological mechanisms related to degrading misfolded proteins in mutant photoreceptors.
\end{abstract}

Key words: photoreceptor; proteasome; proteostasis; retinal degeneration

\section{Significance Statement}

Many mutations linked to inherited retinal degenerations cause the production of misfolded photoreceptor proteins, which overwhelms the capacity of the ubiquitin-proteasome system (UPS) in affected photoreceptors and causes proteostatic stress. This stress has been shown to be a major factor contributing to photoreceptor cell death in multiple mouse models of retinal degeneration. Here, we show that proteostatic stress in two common retinal degeneration models results from insufficient capacities of distinct components of the UPS. These results highlight the complexity of pathophysiological mechanisms related to degrading misfolded proteins in mutant photoreceptors, which must be accounted for in the development of effective strategies to ameliorate these blinding conditions. 


\section{Introduction}

Inherited retinal degenerations affect two to four million people worldwide. These conditions originate from mutations in > 300 genes (https://sph.uth.edu/retnet/), many of which cause protein misfolding in affected photoreceptors. Previous work in multiple mouse models of retinal degeneration has indicated that the requirement to process large amounts of these misfolded proteins causes proteostatic stress, consisting of an insufficient capacity of the ubiquitin-proteasome system (UPS) to degrade proteasome substrates (Lobanova et al., 2013; Liu et al., 2014; Dexter et al., 2018). Conversely, genetic manipulation reducing the proteolytic capacity of proteasomes evokes photoreceptor death in otherwise normal retinas (Ando et al., 2014). It is also argued that proteasomal rather than lysosomal protein degradation is critical for photoreceptor survival in at least one retinal degeneration mouse model (Yao et al., 2018; Qiu et al., 2019). Importantly, ameliorating proteostatic stress by increasing proteasomal activity delayed photoreceptor death in mouse models of retinal degeneration (Lobanova et al., 2018). Despite this progress, the mechanistic understanding of the connection between insufficient UPS function and photoreceptor degeneration remains limited.

Protein degradation by the UPS is typically initiated by polyubiquitination of a target protein substrate, which enables the proteasome to recognize and degrade this protein (Finley, 2009). However, before being degraded by the proteasome, many proteins undergo a preliminary processing step performed by complexes formed by a homo-hexamer of the AAA + ATPase P97 (also known as VCP) and multiple cofactors. P97 complexes are expressed in all cells and participate in a wide range of functions, including protein degradation, membrane fusion, vesicular trafficking, Golgi formation, autophagy, and DNA repair (Meyer and Weihl, 2014; Stach and Freemont, 2017). This versatility originates from the ability of P97 hexamers to associate with various combinations of over 40 interacting cofactors, which confer functional specificity to P97 complexes by regulating substrate in-

Received October 14, 2019; accepted December 4, 2019; First published December 11, 2019.

The authors declare no competing financial interests.

Author contributions: P.M.D., E.S.L., and V.Y.A. designed research; P.M.D. and S.F. performed research; P.M.D. and V.Y.A. analyzed data; P.M.D. and V.Y.A. wrote the paper.

This work was supported by National Institutes of Health (NIH) Grants EY022959 (to V.Y.A.), EY005722 (to V.Y.A.), and EY026468 (to P.M.D.) and by the Unrestricted Award from Research to Prevent Blindness, Inc. to Duke University.

Acknowledgements: We thank Dr. J. Paul Taylor for providing the knock-in mouse overexpressing P97 (the P97oe mouse) and Ms. Y. Hao for preparing the plastic-embedded retinal cross-sections.

E. S. Lobanova's present address: Department of Ophthalmology, University of Florida, Gainesville, FL 32610.

Correspondence should be addressed to Vadim Y. Arshavsky at vadim.arshavsky@duke.edu.

https://doi.org/10.1523/ENEURO.0428-19.2019

Copyright (C) 2020 Dexter et al.

This is an open-access article distributed under the terms of the Creative Commons Attribution 4.0 International license, which permits unrestricted use, distribution and reproduction in any medium provided that the original work is properly attributed. teraction and subcellular localization (Buchberger et al., 2015; Hänzelmann and Schindelin, 2017).

In the context of the UPS, P97 complexes perform several well-established tasks that facilitate the degradation of substrates otherwise inaccessible to proteasomes. First, these complexes participate in extracting unfolded proteins from the endoplasmic reticulum (ER) during ERassociated protein degradation (Bays et al., 2001; Ye et al., 2001; Braun et al., 2002; Jarosch et al., 2002; Rabinovich et al., 2002). Second, they perform partial unfolding of certain polyubiquitinated protein substrates before proteasome entry (Wójcik et al., 2006; Beskow et al., 2009; Blythe et al., 2017; for review, see van den Boom and Meyer, 2018). This may be particularly important for generating the unstructured regions required for initiating protein degradation by proteasomes (Olszewski et al., 2019). Third, P97 complexes can edit polyubiquitin chains on proteins destined for degradation to optimize their proteasomal recognition (Koegl et al., 1999). It has also been hypothesized that P97 complexes may directly bind and feed substrates into the $20 \mathrm{~S}$ proteolytic core of the proteasome, thereby replacing the 19S caps normally performing this function; however, it remains unclear whether such interaction occurs in eukaryotic cells (Barthelme and Sauer, 2012, 2013).

In this study, we investigated whether insufficient UPS function observed in degenerating rod photoreceptors could result, at least in part, from an insufficient cellular capacity for substrate processing by P97 complexes. We examined two complementary models of progressive retinal degeneration: the knock-out mouse lacking the $\gamma$-subunit of the rod $\mathrm{G}$ protein transducin (the $\mathrm{G} \gamma_{1}{ }^{-/-}$ mouse; Lobanova et al., 2008) and the knock-in mouse bearing a single copy of the $\mathrm{P} 23 \mathrm{H}$ mutation in rhodopsin (the P23H mouse; Sakami et al., 2011). Rods of both models were previously documented to suffer from proteostatic stress. In $G \gamma_{1}^{-/-}$mice, this stress results from the production of transducin's $\beta$-subunit $\left(\mathrm{G} \beta_{1}\right)$, which is unable to fold in the absence of $G \gamma_{1}$ (Lobanova et al., 2013). In $\mathrm{P} 23 \mathrm{H}$ mice, mutant rhodopsin misfolds in the ER, causing proteostatic stress (Lobanova et al., 2018) and eventually initiating the unfolded protein response and photoreceptor cell death (Chiang et al., 2015). Importantly, in both models, the associated misfolded protein undergoes highly efficient intracellular degradation and does not accumulate in the affected rods (Lobanova et al., 2008; Sakami et al., 2011).

Previously, proteostatic stress in these mouse models was demonstrated using the proteasomal activity reporter $\mathrm{Ub}^{\mathrm{G} 76 \mathrm{~V}}$-GFP (Dantuma et al., 2000), which accumulated robustly in degenerating $\mathrm{G} \gamma_{1}{ }^{-/-}$and $\mathrm{P} 23 \mathrm{H}$ rods (Lobanova et al., 2013, 2018; Dexter et al., 2018). Because UbG76V GFP degradation by proteasomes requires its partial unfolding by P97 complexes (Wójcik et al., 2006; Beskow

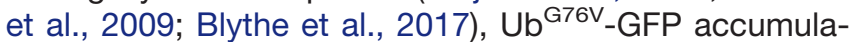
tion in these cells could indicate insufficient capacities of either of these UPS components. Thus, to assess whether the proteostatic stress observed in $\mathrm{G} \gamma_{1}{ }^{-/-}$and $\mathrm{P} 23 \mathrm{H}$ rods may be caused by insufficient P97-dependent substrate processing, we additionally monitored the accumulation 
of an alternative, P97-independent proteasomal activity reporter, oxygen-dependent degradation domainLuciferase (ODDLuc; Safran et al., 2006).

Our analysis revealed a striking difference in the patterns of $\mathrm{Ub}^{\mathrm{G} 76 \mathrm{~V}}$-GFP and ODDLuc reporter accumulation in $\mathrm{G}_{\gamma_{1}^{-1-}}$ and P23H retinas. While retinas of $G \gamma_{1}^{-1-}$ mice exhibited efficient clearance of the P97-independent ODDLuc reporter and accumulation of the P97dependent $\mathrm{Ub}^{\mathrm{G} 76 \mathrm{~V}}$-GFP reporter, both reporters accumulated in the retinas of $\mathrm{P} 23 \mathrm{H}$ mice. These data suggest that the proteostatic stress experienced by these mice likely originates from different pathophysiological mechanisms in which protein degradation by the UPS may or may not be limited by the cellular capacity for P97-dependent substrate processing. We also assessed whether P97 overexpression could ameliorate pathology in $\mathrm{G}_{1}{ }^{-/-}$retinas, in which proteostatic stress appears to result from P97 insufficiency. However, P97 overexpression was toxic to photoreceptors, which greatly complicated the interpretation of the observed phenotype.

Our results highlight the complexity of pathophysiological mechanisms related to degrading misfolded proteins in mutant rods. This complexity must be accounted for in the development of effective strategies to ameliorate these blinding conditions.

\section{Materials and Methods}

\section{Animals}

Mouse care and experiments were performed in accordance with procedures approved by the Institutional Animal Care and Use Committee of Duke University. The Deltagen $\mathrm{G} \gamma_{1}$ knock-out $\left(G \gamma_{1}{ }^{-/}\right)$mouse was licensed from Deltagen, Inc. (Target ID 408) and was previously characterized in (Lobanova et al., 2008, 2013). In this mouse, regions of the $\mathrm{G} \gamma_{1}$ coding sequence (amino acids 17-44 and intron 2) were replaced with a $6.9 \mathrm{~kb}$ IRES-lacZ reporter and neomycin resistance cassette. $\mathrm{P} 23 \mathrm{H}$ rhodopsin mutant knock-in (P23H) mice, characterized in (Sakami et al., 2011), were purchased from The Jackson Laboratory (stock \#017628). Mice heterozygous for the $\mathrm{P} 23 \mathrm{H}$ rhodopsin mutation were used for experiments.

Transgenic mice heterozygously expressing the $\mathrm{Ub}^{\mathrm{G}} 6 \mathrm{~V}$-GFP reporter are described in (Lindsten et al., 2003). Transgenic mice expressing the ODDLuc reporter, characterized in (Safran et al., 2006), were obtained from The Jackson Laboratory (stock \#006206). Both reporters were bred into the $\mathrm{G} \gamma_{1}{ }^{-/-}$and $\mathrm{P} 23 \mathrm{H}$ strains, and reporter experiments were performed using mutant mice heterozygously expressing either reporter. Breeding these mice also required breeding out the $\mathrm{Rd} 1$ mutation that causes severe retinal degeneration from ODDLuc mice. The transgenic mouse overexpressing wild-type (WT) human P97/NCP (the P97oe mouse) was previously characterized in (Custer et al., 2010) and was provided by J. Paul Taylor (St. Jude Children's Research Hospital). Single copies of the Ub ${ }^{\mathrm{G} 76 \mathrm{~V}}$-GFP and P97oe transgenes were bred into the $G \gamma_{1}^{-1-}$ line for $U b^{G 76 V}$-GFP quantification from retinal lysates. Littermates lacking Ub ${ }^{G 76 V}$-GFP expression were used for morphologic analyses.
Transgenic mice were maintained through heterozygous breeding with C57BL/6J WT mice from The Jackson Laboratory (stock \#000664) and tested for the lack of Rd1 and Rd8 mutations. The WT control mice used in Figure $3 B$ were littermates of experimental mice. Non-littermate C57BL/6J WT mice were used in other experiments. Mice of either sex were used for all experiments.

\section{Western blotting}

For Western blot analysis of P97 and Ub ${ }^{\mathrm{G} 76 \mathrm{~V}}$-GFP protein levels in retinal lysates, two mouse retinas per sample were solubilized in $150 \mu \mathrm{l}$ of $1 \%$ Triton X-100 in PBS. Lysates were centrifuged at $16,850 \times g$ for $15 \mathrm{~min}$ at $4^{\circ} \mathrm{C}$, and supernatants were collected. Total protein concentration was measured using the DC Protein Assay kit (Bio-Rad), and samples were diluted with SDS-PAGE sample buffer. Proteins were separated by SDS-PAGE.

\section{Antibodies}

Mouse anti-P97 antibody (ab11433, 1:2000) was from Abcam, rabbit anti-Hsc70 antibody (AD1-SPA-819, $1: 10,000)$ was from Enzo Life Sciences, and mouse antieGFP antibody (632381, 1:5000) was from Clontech. Secondary goat or donkey antibodies conjugated with Alexa Fluor 680 and $800(1: 10,000)$ were from Invitrogen. Protein bands were visualized and quantified using the Odyssey Infrared Imaging System (LI-COR Biosciences).

\section{Luciferase activity assay}

For quantitative analysis of ODDLuc reporter levels, luciferase activity was measured in retinal lysates of threeto four-week-old WT, $G \gamma_{1}^{-/-}$, and P23H mice expressing a single copy of ODDLuc. As a positive control for reporter accumulation, ODDLuc heterozygotes were administered $1 \mu \mathrm{g}$ Roxadustat/FG-4592 (Selleckchem) by intraocular injection, and their retinas were collected $4 \mathrm{~h}$ after injection. For each sample, two retinas were lysed in $150 \mu \mathrm{l}$ ice-cold Glo Lysis buffer (Promega) and homogenized by pulse sonication. Retinal extracts were centrifuged at 500 $\times g$ for 10 min at $4^{\circ} \mathrm{C}$, and the supernatants were centrifuged at $100,000 \times g$ for $1 \mathrm{~h}$ at $4^{\circ} \mathrm{C}$. The supernatants containing the soluble protein fraction were collected for use in the luciferase activity assay and diluted to $1 \mu \mathrm{g} / \mu \mathrm{l}$ with Glo Lysis buffer.

The luciferase activity assay was performed using the Bright-Glo Luciferase Assay System (Promega) according to the manufacturer's protocol. Samples and reagents were thawed to room temperature, and $100 \mu$ l of retinal lysate (containing $100 \mu \mathrm{g}$ of total protein) and $100 \mu \mathrm{l}$ of Bright-Glo reagent were added to each well of a white, flat-bottom 96-well plate and mixed by gentle pipetting. Luminescence was immediately measured at all emission wavelengths $1 \times / \mathrm{min}$ for $20 \mathrm{~min}$ using a SpectraMax M5 luminometer (Molecular Devices) with the following settings: Automix Once; measurement delay $2 \mathrm{~s}$; measurement read $10 \mathrm{~s}$; one read per well. Data were analyzed using the SoftMax Pro 4.8 software to plot relative luminescence units (RLU) as a function of time. For each sample, RLU at time 0 was determined by identifying the $y$-intercept of the line-of-best-fit for the RLU values measured. Data were normalized by dividing experimental 
Table 1. Statistical table

\begin{tabular}{|c|c|c|c|c|}
\hline Line & Figure & Comparison & Type of test & $p$ value \\
\hline a & Fig. $2 A$ & GFP level in $G \gamma_{1}^{-/-}$vs WT & Mann-Whitney & 0.0007 \\
\hline b & Fig. $2 A$ & GFP level in P23H vs WT & Mann-Whitney & 0.0007 \\
\hline C & Fig. $2 A$ & GFP level in $\mathrm{G}_{\gamma_{1}}^{-/-}$vs P23H & Mann-Whitney & 0.0022 \\
\hline d & Fig. $2 B$ & Luc activity in Rox WT vs untreated & Mann-Whitney & 0.0001 \\
\hline e & Fig. $2 B$ & Luc activity in $\mathrm{P} 23 \mathrm{H}$ vs WT & Mann-Whitney & 0.0002 \\
\hline$f$ & Fig. $2 B$ & Luc activity in $G \gamma_{1}^{-/-}$vs WT & Mann-Whitney & $<0.0001$ \\
\hline$g$ & Fig. $2 B$ & Luc activity in $\mathrm{G}_{1}{ }^{-/-}$vs $\mathrm{P} 23 \mathrm{H}$ & Mann-Whitney & 0.0016 \\
\hline $\mathrm{h}$ & Fig. $3 A$ & P97 level in $G \gamma_{1}^{-1-}$ vs WT & Mann-Whitney & 0.8182 \\
\hline i & Fig. $3 A$ & P97 level in P23H vs WT & Mann-Whitney & 0.7056 \\
\hline j & Fig. $3 B$ & P97 level in P97oe vs WT & Mann-Whitney & 0.0079 \\
\hline k & Fig. $3 D$ & Nuclei in P97oe vs WT at three months & Mann-Whitney & 0.0357 \\
\hline | & Fig. $4 B$ & Nuclei in $G \gamma_{1}^{-1-} / \mathrm{P} 970 \mathrm{o}$ vs $G \gamma_{1}^{-/-}$at three months & Mann-Whitney & 0.2 \\
\hline $\mathrm{m}$ & Fig. $4 E$ & Nuclei in $G \gamma_{1}{ }^{-/-} / \mathrm{P} 970 e$ vs $G \gamma_{1}{ }^{-/-}$at six months & Mann-Whitney & 0.0095 \\
\hline $\mathrm{n}$ & Fig. 5 & GFP level in $G \gamma_{1}^{-/-} / P 97 o e$ vs $G \gamma_{1}^{-/-}$ & Mann-Whitney & 0.0079 \\
\hline
\end{tabular}

RLU values by the averaged RLU values obtained from ODDLuc heterozygote control lysates included in each plate.

\section{Histology and microscopy}

Photoreceptor cell loss was evaluated in semi-thin plastic-embedded retinal cross-sections ( $0.5 \mu \mathrm{m}$ thick) obtained from mice at three and six months of age. The retinal cross-sections were prepared as follows: eyes were enucleated from mice and fixed for $1 \mathrm{~h}$ in freshly prepared $2 \%$ formaldehyde with $2.5 \%$ glutaraldehyde in $0.1 \mathrm{M}$ cacodylate buffer containing $2.5 \mathrm{mM} \mathrm{CaCl}_{2}(\mathrm{pH}$ 7.4). The eye globe was then hemisected along the vertical meridian and allowed to fix overnight in the same buffer. The eyecup was rinsed with excess $0.1 \mathrm{M}$ cacodylate buffer $(\mathrm{pH} 7.4)$, placed into $2 \%$ osmium tetroxide for $1.5 \mathrm{~h}$, and rinsed twice with water. The eye cup was gradually dehydrated in an increasing ethanol series (25$100 \%)$ and embedded in Epon; 0.5- $\mu \mathrm{m}$ cross-sections were obtained and stained with toluidine blue for light microscopy. Tiled images of whole retina cross-sections were obtained using the Nikon A1R confocal microscope and aligned and stitched using the NIS-Elements AR software. The number of photoreceptor nuclei in representative segments of outer nuclear layer was counted as a quantitative measure of surviving photoreceptors. Nuclear counts were performed in eight $100-\mu \mathrm{m}$ segments located at $500-\mu \mathrm{m}$ steps from the optic nerve head.

\section{Statistical analysis}

All data are presented as the mean \pm SD. Statistical analyses were performed with GraphPad Prism 7.04. P values are shown in the Statistical Table (Table 1). Results were considered statistically significant when $p<0.05$. Data structure: For all comparisons, $\mathrm{N}$ was too small to determine whether data were normally distributed.

\section{Results}

\section{Comparison of $\mathrm{Ub}^{\mathrm{G} 76 \mathrm{~V}}$-GFP and ODDLuc accumulation in $\mathbf{G} \gamma_{1}{ }^{-/-}$and $\mathrm{P} 23 \mathrm{H}$ retinas}

We first assessed P97-dependent UPS substrate processing in $\mathrm{G}_{1}{ }^{-/-}$and $\mathrm{P} 23 \mathrm{H}$ mice by monitoring the retinal accumulation of two in vivo proteasomal activity reporters whose degradation is either P97-dependent or P97independent (Fig. 1). The first reporter, Ub G76V_GFP (Dantuma et al., 2000), is degraded by proteasomes following its partial unfolding by the P97-Ufd1-Npl4 substrateprocessing complex (Wójcik et al., 2006; Beskow et al., 2009; Blythe et al., 2017). The second reporter, ODDLuc (Safran et al., 2006), is efficiently degraded by proteasomes without P97 involvement (Chou and Deshaies, 2011). The P97 independence of ODDLuc was previously established using a cell line expressing both Ub ${ }^{G 76 V}$-GFP and ODDLuc. Treatment of these cells with proteasome inhibitor blocked the degradation of both reporters. On the other hand, P97 inhibition or its siRNA knock-down blocked the degradation of Ub G76V - GFP but not ODDLuc (Chou and Deshaies, 2011).

We bred $\mathrm{G}_{1}{ }^{-/-}$and $\mathrm{P} 23 \mathrm{H}$ mice with the mice ubiquitously expressing either Ub ${ }^{G 76 V}$-GFP (Lindsten et al., 2003) or ODDLuc (Safran et al., 2006) and assessed the accumulation of each reporter in the retinas of three- to

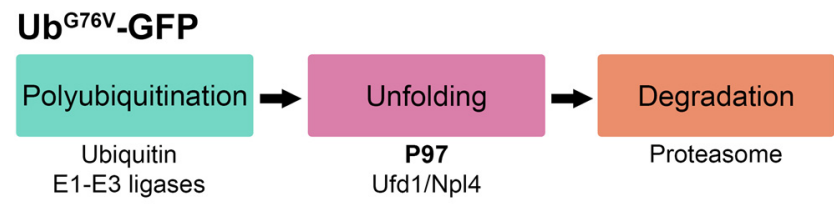

ODDLuc

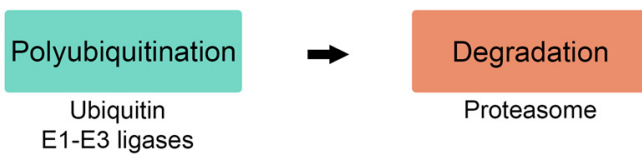

Figure 1. The in vivo proteasomal activity reporters $\mathrm{Ub}^{\mathrm{G} 76 \mathrm{~V}}$-GFP and ODDLuc are degraded through P97-dependent and P97independent mechanisms. Top, Ub ${ }^{G 76 V}$-GFP is a fusion protein consisting of GFP fused to a non-cleavable molecule of ubiquitin, which acts as a constitutively active degradation signal. $\mathrm{Ub}^{\mathrm{G} 76 \mathrm{~V}}$-GFP is degraded by proteasomes following its polyubiquitination and partial unfolding by the P97-Ufd1-Npl4 complex. Bottom, ODDLuc is a fusion protein consisting of firefly luciferase fused to an alternative degradation signal, the ODD of HIF1 $\alpha$. This reporter is polyubiquitinated and degraded by proteasomes without P97 processing. Conditions of inhibited, impaired, or insufficient UPS function result in the intracellular accumulation of either reporter. 
A

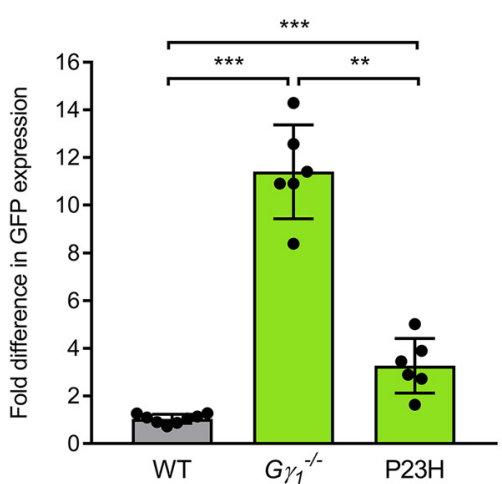

B

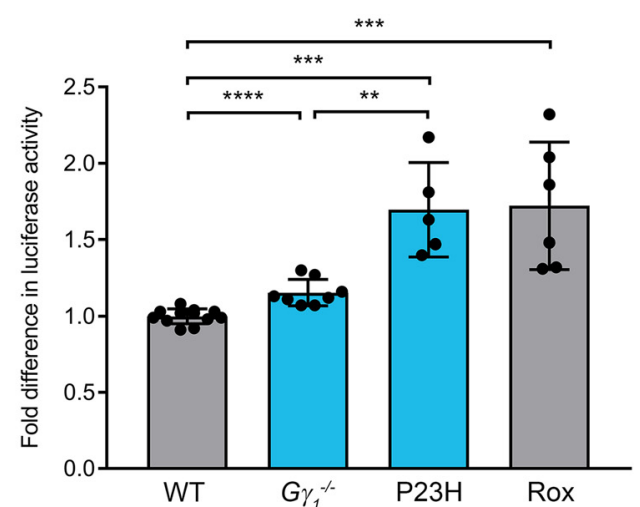

Figure 2. Comparative analysis of proteasomal activity reporter accumulation in $\mathrm{G}_{1}{ }^{-/-}$and $\mathrm{P} 23 \mathrm{H}$ retinas. $\boldsymbol{A}$, The $\mathrm{Ub}^{\mathrm{G} 76 \mathrm{~V}}-\mathrm{GFP}$ reporter was detected in retinal lysates ( $25 \mu \mathrm{g}$ total protein/lane) of three- to four-week-old $\mathrm{G} \gamma_{1}{ }^{-/-}$or $\mathrm{P} 23 \mathrm{H}$ mice by Western blotting with an anti-GFP antibody; the densities of the $\mathrm{Ub}^{\mathrm{G} 76 \mathrm{~V}}$-GFP bands were normalized to the Hsc70 loading control. The number of mice analyzed was the following: WT, 8; $\mathrm{G}_{1}{ }^{-/-}, 6 ; \mathrm{P} 23 \mathrm{H}, 6$. Each dot represents a single data point. $\boldsymbol{B}$, The ODDLuc reporter was detected in retinal lysates (100 $\mathrm{g}$ total protein/reaction) from three- to four-week-old $\mathrm{G} \gamma_{1}{ }^{-/-}$or P23H mice using the Bright-Glo Luciferase Assay system. Retinal lysates from ODDLuc mice treated with the HIF prolyl hydroxylase inhibitor Roxadustat (Rox) were used as a positive control for reporter accumulation. The number of mice analyzed was the following: WT, 12; G $\gamma_{1}^{-/-}, 8 ; \mathrm{P} 23 \mathrm{H}, 5 ; \mathrm{Rox}, 6$. Each dot represents a single data point. Data are shown as the mean \pm SD. $* * p<0.01, * * * p<0.001, * * * * p<0.0001$.

four-week-old mice, in which photoreceptor degeneration has just begun. As reported previously (Lobanova et al., 2013, 2018; Dexter et al., 2018), UbG76V-GFP accumulated in retinas of both $\mathrm{G} \gamma_{1}{ }^{-1-}$ and $\mathrm{P} 23 \mathrm{H}$ mice (Fig. $2 A$ ). Retinal $\mathrm{Ub}^{\mathrm{G} 76 \mathrm{~V}}$-GFP content increased $11.5 \pm 2.0$-fold $(p$ $=0.0007)$ in $\mathrm{G}_{1}{ }^{-/-}$and $3.3 \pm 1.2$-fold $(p=0.0007)$ in $\mathrm{P} 23 \mathrm{H}$ mice relative to WT controls. ${ }^{\mathrm{a}, \mathrm{b}}$

The accumulation of ODDLuc in retinal lysates of $G \gamma_{1}{ }^{-/}$and $\mathrm{P} 23 \mathrm{H}$ mice was monitored using the luciferase activity assay. As a positive control for ODDLuc accumulation, we injected the HIF prolyl hydroxylase inhibitor Roxadustat/FG-4592 in the vitreous of ODDLuc heterozygotes and collected their retinas 4 $\mathrm{h}$ after injection. This treatment caused an increase in ODDLuc accumulation of $1.7 \pm 0.4$-fold $(p=0.0001$; Fig. $2 B)$, ${ }^{d}$ which we considered as a benchmark of this reporter's sensitivity. Notably, this signal was smaller than the signal produced by $\mathrm{Ub}^{\mathrm{G} 76 \mathrm{~V}}$-GFP, yet sufficiently significant for quantitative analysis.

We next analyzed ODDLuc accumulation in the retinas of mutant mice (Fig. 2B). ODDLuc accumulation in $\mathrm{P} 23 \mathrm{H}$ retinas increased $1.7 \pm 0.3$-fold $(p=0.0002)$, reaching the same level as observed in the Roxadustat control. ${ }^{e}$ On the other hand, ODDLuc accumulation in $G \gamma_{1}{ }^{-/-}$retinas increased by only $1.2 \pm 0.1$-fold $(p<0.0001) .{ }^{f}$ Notably, this pattern was qualitatively opposite to the pattern of $\mathrm{Ub}^{\mathrm{G} 76 \mathrm{~V}}$-GFP accumulation, which was higher in $\mathrm{G} \gamma_{1}^{-/-}$ retinas. This difference suggests that, though both $G \gamma_{1}^{-1-}$ and $\mathrm{P} 23 \mathrm{H}$ rods experience proteostatic stress, this condition likely results from insufficient capacities of different UPS components. The relatively high level of ODDLuc accumulation in $\mathrm{P} 23 \mathrm{H}$ retinas suggests that UPS function in their rods is limited by an insufficient cellular capacity for proteasomal degradation. On the other hand, the combination of a high level of $\mathrm{Ub}^{\mathrm{G} 76 \mathrm{~V}}$-GFP accumulation and a low level of ODDLuc accumulation in $G \gamma_{1}^{-/-}$retinas suggests that UPS function in this model may be limited by P97-dependent substrate processing. The latter could not be explained merely by a reduction in the expression of P97, as P97 expression levels were essentially indistinguishable from WT and identical in the retinas of both mouse models (Fig. $3 A)^{\text {h, }}$,

\section{Characterization of the retinal morphology of the P97-overexpressing mouse}

We next followed up on our observation that proteostatic stress in $\mathrm{G}_{1}{ }^{-1-}$ retinas may result from insufficient P97-dependent substrate processing. Currently, there are no available strategies for increasing the substrateprocessing capacity of $\mathrm{P} 97$ complexes in an in vivo context. We attempted to accomplish this through the most straightforward strategy of overexpressing P97 itself. For this purpose, we obtained the transgenic mouse line ubiquitously overexpressing WT human P97 (the P97oe mouse), described in Custer et al. (2010). The P97oe mouse was characterized as phenotypically normal, with no change in development, weight, or survival compared to WT mice. However, the retinal phenotype of this mouse was not assessed.

We first determined the P97 content in P97oe retinas (Fig. 3B). Quantitative Western blotting of retinal lysates from one-month-old mice indicated a robust, $2.4 \pm 0.4$ fold increase in their P97 content $(p=0.0079) .{ }^{j}$ This overexpression level was essentially the same as previously reported for the brain (Custer et al., 2010). We next investigated whether this increase in P97 expression affects photoreceptor health by analyzing the retinal morphology of P97oe mice in thin plastic sections from threemonth-old mice (Fig. 3C-E). To quantify the effect of P97 overexpression on photoreceptor survival, we counted the number of photoreceptor nuclei in $100-\mu \mathrm{m}$ retinal segments at different distances from the optic nerve and plotted the results as a spider diagram (Fig. $3 C$ ). We found that P97oe retinas exhibited marked photoreceptor loss at all analyzed regions of the retina, with the total nuclear count obtained from these regions decreasing by $\sim 31 \%$ 

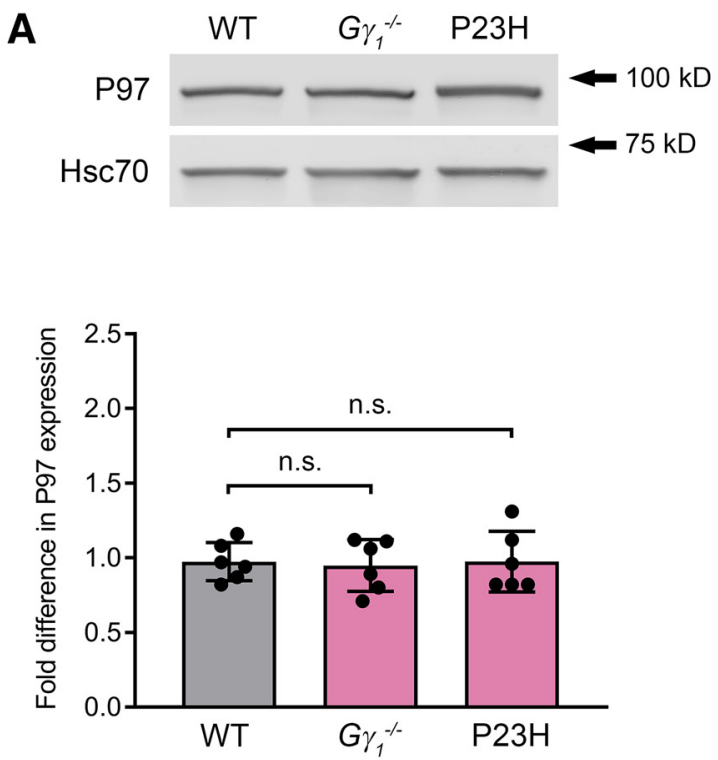

C

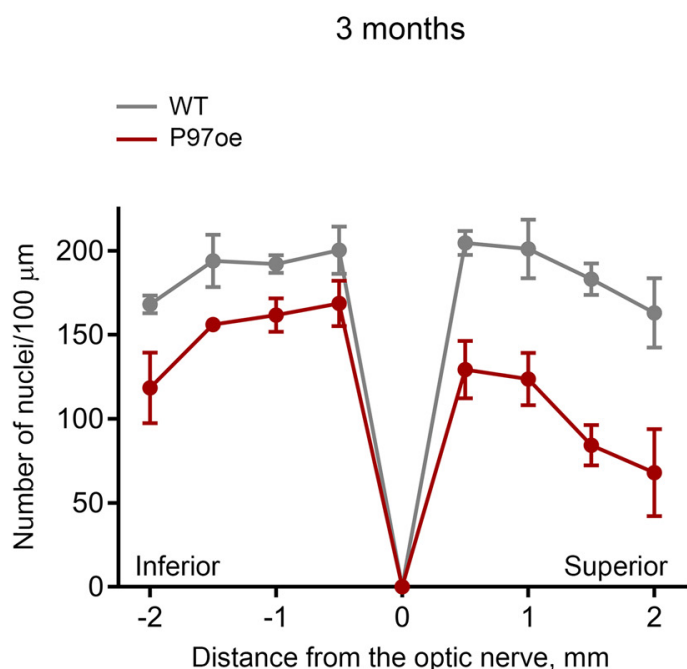

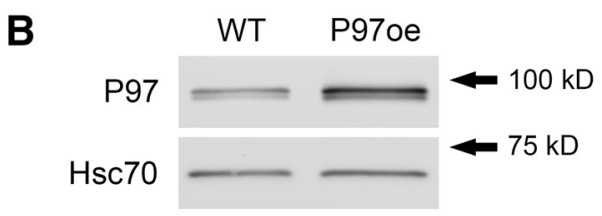

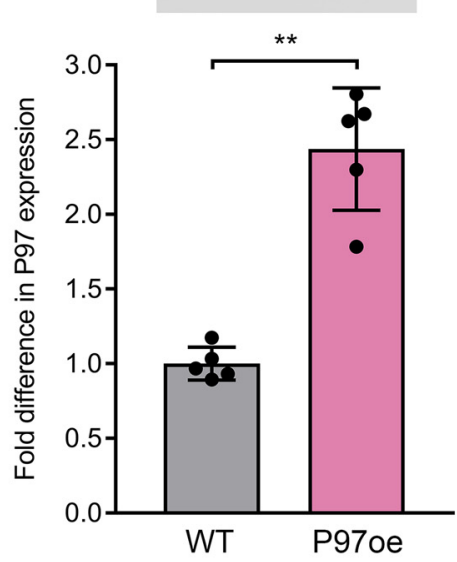

E

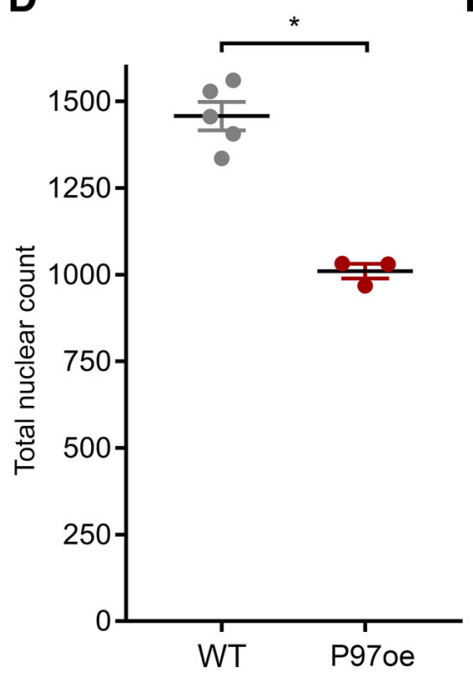

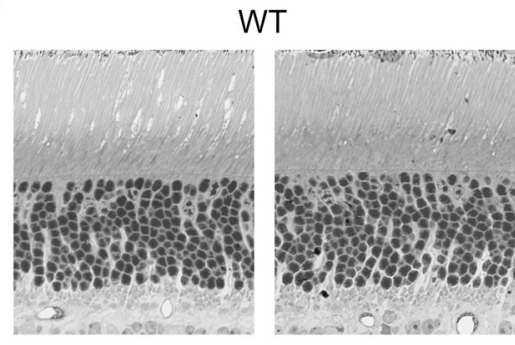

P97oe

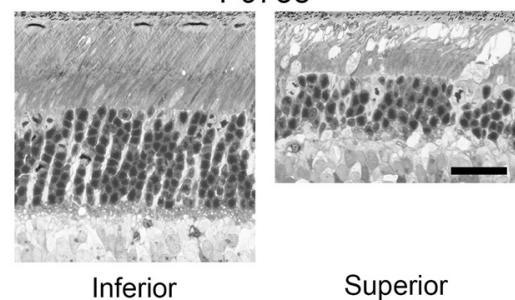

Figure 3. Overexpression of P97 affects photoreceptor survival. A, P97 protein level was detected in retinal lysates $(2 \mu \mathrm{g}$ total protein/lane) of one-month-old $\mathrm{G}_{1}{ }^{-/-}$or $\mathrm{P} 23 \mathrm{H}$ mice by Western blotting with an anti-P97 antibody; the densities of the P97 bands were normalized to the Hsc70 loading control. The number of mice analyzed was the following: WT, 6; G $\gamma_{1}{ }^{-1-}, 6$; P23H, 6. Each dot represents a single data point. $\boldsymbol{B}, \mathrm{P} 97$ protein level was detected in retinal lysates $(2 \mu \mathrm{g}$ total protein/lane) from one-month-old WT and P97-overexpressing (P97oe) mice as in $\boldsymbol{A}$; Hsc70 was used as a loading control. The number of mice analyzed was the following: WT, 5; P97oe, 5. Each dot represents a single data point. $\boldsymbol{C}$, Spider diagrams representing the number of photoreceptor nuclei in $100-\mu \mathrm{m}$ segments of the inferior and superior retina at various distances from the optic nerve head. The number of mice analyzed was the following: WT, 5; P97oe, 3. Mice were three months old. $\boldsymbol{D}$, The total number of nuclei in all eight 100- $\mu \mathrm{m}$ retinal segments represented in spider diagrams of panel $\boldsymbol{C}$. Each dot represents a single data point. $\boldsymbol{E}$, Representative images of inferior and superior retina cross-sections from WT and P97oe at the 1-mm distance from the optic nerve head. Mice were three months old. Scale bar $=25 \mu \mathrm{m}$. Data are shown as mean \pm SD. n.s. indicates $p>0.05 . * p<0.05, * * p<0.01$.

compared to WT controls (from $1458 \pm 91$ nuclei in WT to $1010 \pm 36$ in P97oe, $p=0.0357$; Fig. 3D). ${ }^{k}$ We also analyzed retinas from P97oe mice at six months of age and observed $\sim 42 \%$ photoreceptor loss compared to WT (data not shown).

These data indicate that, although P97oe mice were not previously reported to display any obvious neurologic abnormalities (Custer et al., 2010), the $\sim 2$-fold increase in retinal P97 content in these mice is toxic to rod photore- ceptors and causes significant cell death before three months of age. Additionally, we observed that that the toxic effect of P97 overexpression was more pronounced in the superior retina than in the inferior retina (Fig. $3 C, E$ ).

\section{Photoreceptor degeneration in $\mathrm{G} \gamma_{1}{ }^{-/-}$mice overexpressing P97}

Despite observing some P97 overexpression toxicity, we nonetheless decided to evaluate whether P97 overex- 
A

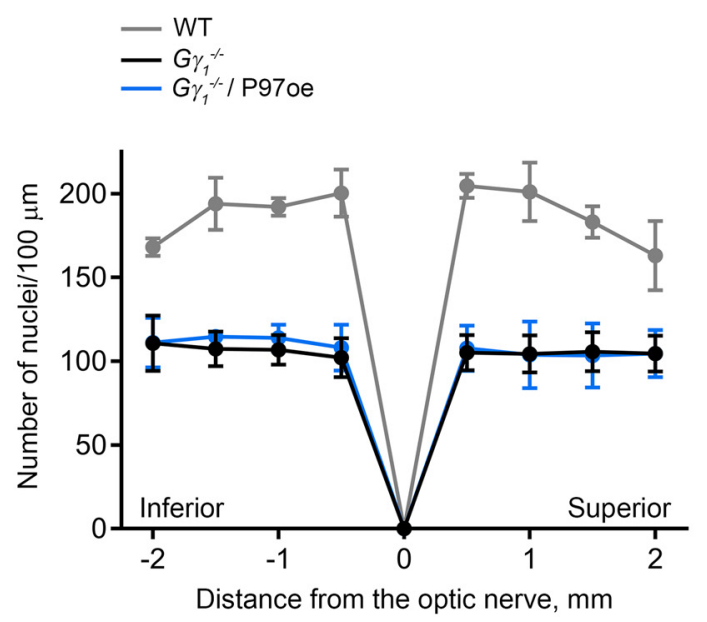

D

6 months

$$
\begin{aligned}
& -W T \\
& -G \gamma_{1}{ }^{-1} \\
& -G \gamma_{1}{ }^{-1 / P 97 o e}
\end{aligned}
$$

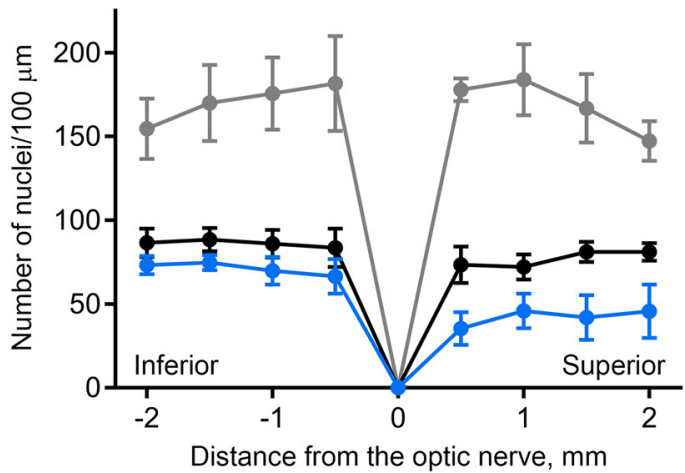

B

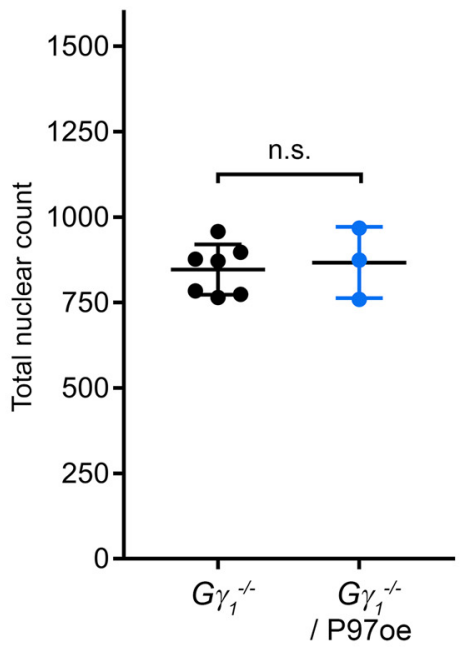

E

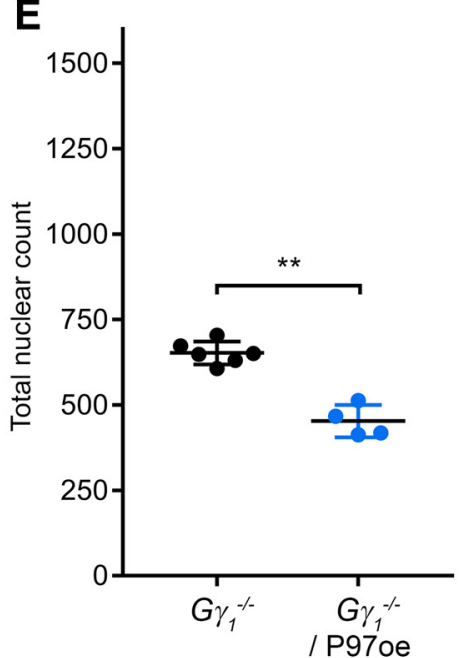

C
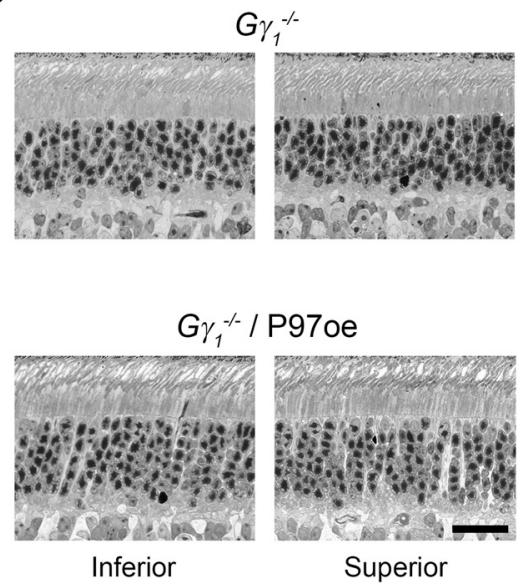

F
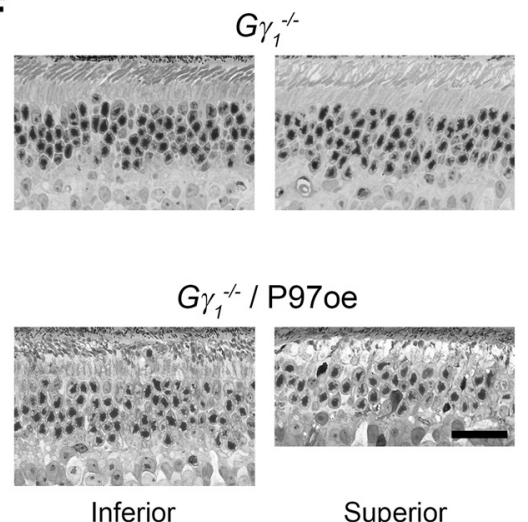

Figure 4. Overexpression of P97 affects photoreceptor survival in $G \gamma_{1}^{-/-}$mice. $\boldsymbol{A}-\boldsymbol{F}$, Morphology of $G \gamma_{1}{ }^{-/-}$retinas with or without P97 overexpression at three $(\boldsymbol{A}-\boldsymbol{C})$ or six $(\boldsymbol{D}-\boldsymbol{F})$ months of age. $\boldsymbol{A}, \boldsymbol{D}$, Spider diagrams representing the number of photoreceptor nuclei in $100-\mu \mathrm{m}$ segments of the inferior and superior retina at various distances from the optic nerve head. The number of mice analyzed at three months of age was the following: WT, 3; $G \gamma_{1}{ }^{-/-}, 7 ; G \gamma_{1}{ }^{-/-} /$P97oe, 3. The number of mice analyzed at six months of age was the following: WT, 4; $G \gamma_{1}^{-1-}, 6 ; G \gamma_{1}{ }^{-/-} / \mathrm{P} 970 e, 4 . \boldsymbol{B}, \boldsymbol{E}$, The total number of nuclei in all eight 100- $\mu \mathrm{m}$ retinal segments represented in panels $\boldsymbol{A}, \boldsymbol{D}$. C, $\boldsymbol{F}$, Representative images of inferior and superior retina cross-sections at the 1-mm distance from the optic nerve head. Scale bar $=25 \mu \mathrm{m}$. Data are shown as mean \pm SD. n.s. indicates $p>0.05 . * * p<0.01$.

pression may improve photoreceptor survival in $G \gamma_{1}{ }^{-/}$, which apparently suffers from P97 insufficiency. We bred the $G \gamma_{1}^{-1-}$ mouse with the P97oe mouse and assessed photoreceptor survival in the resulting line at three and six months of age (Fig. 4). At three months of age, $G \gamma_{1}^{-/-}$and $G \gamma_{1}^{-1 /} /$ P97oe retinas exhibited a nearly identical degree of photoreceptor loss (Fig. 4A-C).' By six months of age, $\mathrm{G} \gamma_{1}{ }^{-/} / \mathrm{P} 970 \mathrm{oe}$ retinas exhibited a slight increase in photoreceptor loss compared to $G \gamma_{1}^{-1-}$ retinas (453 \pm 47 in $G \gamma_{1}{ }^{-/ /} /$P97oe vs $652 \pm 34$ nuclei in $G \gamma_{1}^{-/}, p=0.0095$; Fig. $4 D-F){ }^{\mathrm{m}}$ Once again, the superior retina was affected to a larger degree than the inferior retina. Thus, P97 overexpression only exacerbated the deleterious effects of $\mathrm{G} \gamma_{1}$ knock-out.

\section{Ub ${ }^{\mathrm{G} 76 \mathrm{~V}}$-GFP accumulation in $\mathrm{G} \gamma_{1}{ }^{-/-}$retinas overexpressing P97}

Finally, we investigated whether P97 overexpression actually facilitated P97-dependent substrate processing in $\mathrm{G}_{1}{ }_{1}^{-/-}$photoreceptors. To do this, we bred single copies of the P97 and $\mathrm{Ub}^{\mathrm{G} 76 \mathrm{~V}}$-GFP transgenes into the $\mathrm{G} \gamma_{1}{ }^{-/-}$line. Ub ${ }^{\mathrm{G} 76 \mathrm{~V}}$-GFP reporter accumulation in the resulting mouse was quantified by Western blotting of retinal lysates at one month of age. Contrary to our expectations, retinas of $\mathrm{G} \gamma_{1}^{-/ /} /$P97oe mice exhibited a slight but statistically significant $(1.5 \pm 0.2$-fold; $p=$ 0.0079 ) increase in $\mathrm{Ub}^{\mathrm{G} 76 \mathrm{~V}}$-GFP accumulation compared to $\mathrm{G} \gamma_{1}^{-1-}$ (Fig. 5). ${ }^{\mathrm{n}}$ This result indicates that P97 overexpression increases the level of proteostatic stress already experienced by these mice. 

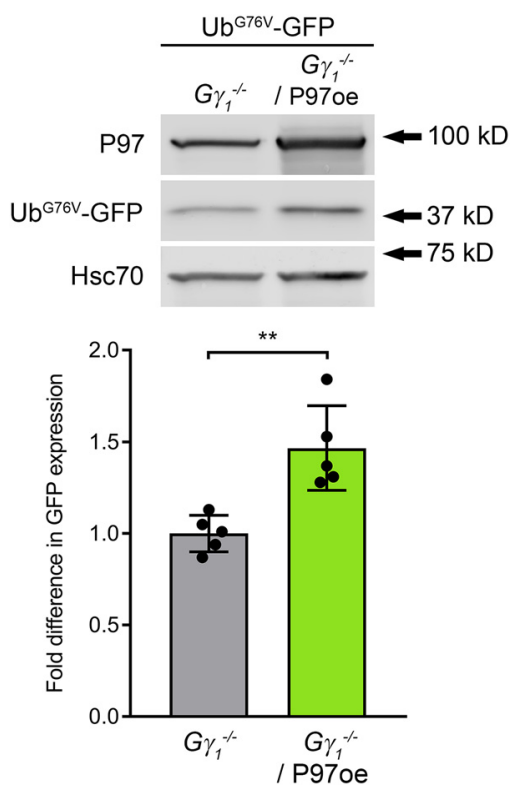

Figure 5. Overexpression of P97 causes increased accumulation of the $\mathrm{Ub}^{\mathrm{G} 76 \mathrm{~V}}$-GFP reporter. The Ub ${ }^{\mathrm{G} 76 V_{-}}$-GFP reporter was detected in retinal lysates (25 $\mu \mathrm{g}$ total protein/lane) of one-monthold $G \gamma_{1}{ }^{-/-}$and $G \gamma_{1}{ }^{-/-} /$P97oe mice by Western blotting with an anti-GFP antibody; the densities of the Ub ${ }^{\text {G76V }}$-GFP bands were normalized to the Hsc70 loading control. The number of mice analyzed was the following: $\mathrm{G} \gamma_{1}{ }^{-/-}, 5 ; \mathrm{G} \gamma_{1}{ }^{-/-} / \mathrm{P} 970 \mathrm{o}, 5$. Each dot represents a single data point. Data are shown as the mean \pm SD. $* * p<0.01$

\section{Discussion}

Recent work has established that proteostatic stress, consisting of an insufficient capacity of the UPS to degrade proteasomal substrates, is a major contributing factor in multiple forms of inherited retinal degeneration (Lobanova et al., 2013; Liu et al., 2014; Dexter et al., 2018). In this study, we evaluated whether this proteostatic stress may originate from insufficient P97dependent substrate processing, which is required for the proteasomal degradation of a large number of UPS targets. Our data indicate that this could be the case, although only for a subset of mutations associated with photoreceptor protein misfolding. Whereas insufficient UPS function in $G \gamma_{1}{ }^{-/-}$rods appears to result from insufficient P97-dependent substrate processing, proteasomal degradation itself appears to limit UPS function in $\mathrm{P} 23 \mathrm{H}$ rods. These results argue that individual mutations must be considered when developing therapeutic interventions for reducing proteostatic stress in these blinding conditions.

Whereas the exact mechanistic explanation for the difference in the rate-limiting UPS components between these mutant mouse models remains to be addressed experimentally, we can offer the following hypothetical explanation consistent with our current results. Photoreceptors are known to constantly renew their light-sensitive membranes, which requires continuous biosynthesis of unusually large amounts of membrane proteins. This implies that they have a high capacity of the ER-associated degradation machin- ery, including P97 complexes, to perform quality control of proteins produced in the ER. One can speculate that this capacity is naturally so high that it is sufficient to process misfolded $\mathrm{P} 23 \mathrm{H}$ rhodopsin molecules. However, these cells do not appear to have enough downstream proteasomes, ultimately leading to proteostatic stress in $\mathrm{P} 23 \mathrm{H}$ mice. The cytoplasmic pool of P97 in photoreceptors may be more trivial and easily saturable by the large amounts of uncoupled $\mathrm{G} \beta_{1}$ produced in $\mathrm{G} \gamma_{1}{ }^{-/-}$rods. Assuming that these cells lack the ability to redirect the ER-associated P97 pool to the cytoplasm, the proteostatic stress in $G \gamma_{1}{ }^{-/-}$photoreceptors arises from P97 insufficiency.

Interestingly, the most straightforward approach to overcoming insufficient P97-dependent substrate processing in $\mathrm{G}_{1}{ }^{-/-}$photoreceptors, consisting of overexpressing this protein, was not productive. In fact, P97 overexpression caused progressive death of otherwise normal photoreceptors. Furthermore, P97 overexpression exacerbated proteostatic stress and accelerated photoreceptor loss in $G \gamma_{1}^{-/-}$mice. The simplest explanation for these effects is that increased cellular content of P97 does not automatically lead to formation of additional functional P97 complexes and may instead impose its own stress on the UPS. However, recent studies of P97 mutations linked to human disease suggest a more complex explanation for the photoreceptor cell death we observe in P97oe mice.

Mutations in $\mathrm{P} 97$ can cause multisystem proteinopathy (MSP), a syndromic disorder affecting the brain, bone and muscle. Disease-associated mutations in P97 were recently shown to increase the rate of substrate unfolding by P97 complexes in vitro (Blythe et al., 2017, 2019), and expression of such 'overactive' P97 mutants in Drosophila and mice was highly toxic (Custer et al., 2010; Ritson et al., 2010; Zhang et al., 2017). These data raise a possibility that the toxicity observed in P97overexpressing photoreceptors may result from a pathologic increase in P97-dependent substrate processing. While this possibility is not immediately supported by our finding that P97 overexpression increases Ub $\mathrm{UbV}_{\text {-GFP }}$ accumulation in $\mathrm{G}_{1}{ }^{-/-}$retinas, cell death in P97overexpressing rods may arise from multiple contributing factors. For example, increased P97-dependent processing and degradation of regulatory proteins could inappropriately activate critical signaling pathways, such as $\mathrm{NF}-\kappa \mathrm{B}$ (Custer et al., 2010), leading to cellular dysfunction and cell death. Such perturbations could not be detected by merely monitoring cytosolic Ub ${ }^{\text {G76V }}$-GFP levels. Given the complexity of this system, understanding how to finetune P97-dependent substrate processing to alleviate the proteostatic stress caused by misfolded protein production will require a broader consideration of the entire P97-interacting network.

Curiously, photoreceptors are the only cells studied so far that are negatively affected by P97 overexpression. In the initial characterization of the P97oe mouse (Custer et al., 2010), these mice developed normally and displayed no abnormalities in weight or survival at all ages evaluated (up to 15 months of age). Kidney, liver, spleen, intestine, and gastrocnemius and quadriceps muscles of 
P97oe mice appeared histologically normal, despite confirmed transgene expression in these tissues. Brains harvested from P97oe mice, in which P97 expression was increased $\sim 2$-fold, revealed no overt signs of degeneration by hematoxylin and eosin staining. P97oe mice also exhibited normal hind-limb clasping, a behavior which is disrupted in numerous mouse models of neurodegeneration and is a common indicator of central nervous system pathology (Reddy et al., 1999). Finally, tests of declarative memory and anxiety-associated behaviors also revealed no abnormalities in P97oe mice (Custer et al., 2010). Given the otherwise healthy phenotype of the P97oe mouse, the toxicity we observed in P97oe photoreceptors came as a surprise to us. However, what could be viewed as even more surprising is that only one cell type is negatively affected by 2- to 3-fold overexpression of P97, which is already one of the most abundantly-expressed proteins in the cell (Peters et al., 1990).

Finally, our new data make a mechanistic connection with a recent study in which we demonstrated that overexpression of the PA28 $\alpha$ subunit of the $11 \mathrm{~S}$ proteasome cap is therapeutic (Lobanova et al., 2018). In this study, we showed that PA28 $\alpha$ overexpression increases proteasomal activity and delays photoreceptor cell loss in both $\mathrm{G} \gamma_{1}{ }^{-/-}$and $\mathrm{P} 23 \mathrm{H}$ mice. One observation that we could not immediately explain was that photoreceptor protection on proteasomal activation was much more pronounced in P23H mice. However, a plausible explanation can now be offered in light of our current findings. Our data suggest that the proteostatic stress in $G \gamma_{1}^{-1-}$ rods results from an insufficient cellular capacity for P97-dependent substrate processing, whereas $\mathrm{P} 23 \mathrm{H}$ rods experience insufficient proteasomal degradation. Therefore, it is entirely expected that direct proteasomal activation would be more effective in $\mathrm{P} 23 \mathrm{H}$ rods, whereas protection of $\mathrm{G} \gamma_{1}{ }^{-/-}$rods would require increasing substrate processing by P97 complexes.

\section{References}

Ando R, Noda K, Tomaru U, Kamoshita M, Ozawa Y, Notomi S, Hisatomi T, Noda M, Kanda A, Ishibashi T, Kasahara M, Ishida S (2014) Decreased proteasomal activity causes photoreceptor degeneration in mice. Invest Ophthalmol Vis Sci 55:4682-4690.

Barthelme D, Sauer RT (2012) Identification of the Cdc48.20S proteasome as an ancient AAA+ proteolytic machine. Science 337: 843-846.

Barthelme D, Sauer RT (2013) Bipartite determinants mediate an evolutionarily conserved interaction between Cdc48 and the $20 \mathrm{~S}$ peptidase. Proc Natl Acad Sci USA 110:3327-3332.

Bays NW, Wilhovsky SK, Goradia A, Hodgkiss-Harlow K, Hampton RY (2001) HRD4/NPL4 is required for the proteasomal processing of ubiquitinated ER proteins. Mol Biol Cell 12:4114-4128.

Beskow A, Grimberg KB, Bott LC, Salomons FA, Dantuma NP, Young $P$ (2009) A conserved unfoldase activity for the p97 AAAATPase in proteasomal degradation. J Mol Biol 394:732-746.

Blythe EE, Olson KC, Chau V, Deshaies RJ (2017) Ubiquitin- and ATP-dependent unfoldase activity of P97/NCP.NPLOC4.UFD1L is enhanced by a mutation that causes multisystem proteinopathy. Proc Natl Acad Sci USA 114:E4380-E4388.

Blythe EE, Gates SN, Deshaies RJ, Martin A (2019) Multisystem proteinopathy mutations in VCP/p97 increase NPLOC4.UFD1L binding and substrate processing. Structure 27:1820-1829.e4.
Braun S, Matuschewski K, Rape M, Thoms S, Jentsch S (2002) Role of the ubiquitin-selective CDC48(UFD1/NPL4)chaperone (segregase) in ERAD of OLE1 and other substrates. EMBO J 21:615-621.

Buchberger A, Schindelin H, Hänzelmann P (2015) Control of p97 function by cofactor binding. FEBS Lett 589:2578-2589.

Chiang WC, Kroeger H, Sakami S, Messah C, Yasumura D, Matthes MT, Coppinger JA, Palczewski K, LaVail MM, Lin JH (2015) Robust endoplasmic reticulum-associated degradation of rhodopsin precedes retinal degeneration. Mol Neurobiol 52:679-695.

Chou TF, Deshaies RJ (2011) Quantitative cell-based protein degradation assays to identify and classify drugs that target the ubiquitin-proteasome system. J Biol Chem 286:16546-16554.

Custer SK, Neumann M, Lu H, Wright AC, Taylor JP (2010) Transgenic mice expressing mutant forms VCP/p97 recapitulate the full spectrum of IBMPFD including degeneration in muscle, brain and bone. Hum Mol Genet 19:1741-1755.

Dantuma NP, Lindsten K, Glas R, Jellne M, Masucci MG (2000) Short-lived green fluorescent proteins for quantifying ubiquitin/ proteasome-dependent proteolysis in living cells. Nat Biotechnol 18:538-543.

Dexter PM, Lobanova ES, Finkelstein S, Spencer WJ, Skiba NP, Arshavsky VY (2018) Transducin $\beta$-subunit can interact with multiple G-protein $\gamma$-subunits to enable light detection by rod photoreceptors. eNeuro 5.

Finley D (2009) Recognition and processing of ubiquitin-protein conjugates by the proteasome. Annu Rev Biochem 78:477-513.

Hänzelmann P, Schindelin H (2017) The interplay of cofactor interactions and post-translational modifications in the regulation of the AAA+ ATPase p97. Front Mol Biosci 4:21.

Jarosch E, Taxis C, Volkwein C, Bordallo J, Finley D, Wolf DH, Sommer T (2002) Protein dislocation from the ER requires polyubiquitination and the AAA-ATPase Cdc48. Nat Cell Biol 4:134139.

Koegl M, Hoppe T, Schlenker S, Ulrich HD, Mayer TU, Jentsch S (1999) A novel ubiquitination factor, E4, is involved in multiubiquitin chain assembly. Cell 96:635-644.

Lindsten K, Menéndez-Benito V, Masucci MG, Dantuma NP (2003) A transgenic mouse model of the ubiquitin/proteasome system. Nat Biotechnol 21:897-902.

Liu YP, Tsai IC, Morleo M, Oh EC, Leitch CC, Massa F, Lee BH, Parker DS, Finley D, Zaghloul NA, Franco B, Katsanis N (2014) Ciliopathy proteins regulate paracrine signaling by modulating proteasomal degradation of mediators. J Clin Invest 124:20592070.

Lobanova ES, Finkelstein S, Herrmann R, Chen YM, Kessler C, Michaud NA, Trieu LH, Strissel KJ, Burns ME, Arshavsky VY (2008) Transducin $\gamma$-subunit sets expression levels of $\alpha$ - and $\beta$-subunits and is crucial for rod viability. J Neurosci 28:3510-3520.

Lobanova ES, Finkelstein S, Skiba NP, Arshavsky VY (2013) Proteasome overload is a common stress factor in multiple forms of inherited retinal degeneration. Proc Natl Acad Sci USA 110:99869991.

Lobanova ES, Finkelstein S, Li J, Travis AM, Hao Y, Klingeborn M, Skiba NP, Deshaies RJ, Arshavsky VY (2018) Increased proteasomal activity supports photoreceptor survival in inherited retinal degeneration. Nat Commun 9:1738.

Meyer H, Weihl CC (2014) The VCP/p97 system at a glance: connecting cellular function to disease pathogenesis. J Cell Sci 127: 3877-3883.

Olszewski MM, Williams C, Dong KC, Martin A (2019) The Cdc48 unfoldase prepares well-folded protein substrates for degradation by the $26 \mathrm{~S}$ proteasome. Commun Biol 2:29.

Peters JM, Walsh MJ, Franke WW (1990) An abundant and ubiquitous homo-oligomeric ring-shaped ATPase particle related to the putative vesicle fusion proteins Sec18p and NSF. EMBO J 9:17571767.

Qiu Y, Yao J, Jia L, Thompson DA, Zacks DN (2019) Shifting the balance of autophagy and proteasome activation reduces proteotoxic cell death: a novel therapeutic approach for restoring photoreceptor homeostasis. Cell Death Dis 10:547. 
Rabinovich E, Kerem A, Fröhlich KU, Diamant N, Bar-Nun S (2002) AAA-ATPase p97/Cdc48p, a cytosolic chaperone required for endoplasmic reticulum-associated protein degradation. Mol Cell Biol 22:626-634.

Reddy PH, Charles V, Williams M, Miller G, Whetsell WOJ, Tagle DA (1999) Transgenic mice expressing mutated full-length HD cDNA: a paradigm for locomotor changes and selective neuronal loss in Huntington's disease. Philos Trans R Soc Lond B Biol Sci 354: 1035-1045.

Ritson GP, Custer SK, Freibaum BD, Guinto JB, Geffel D, Moore J, Tang W, Winton MJ, Neumann M, Trojanowski JQ, Lee VM, Forman MS, Taylor JP (2010) TDP-43 mediates degeneration in a novel Drosophila model of disease caused by mutations in VCP/ p97. J Neurosci 30:7729-7739.

Safran M, Kim WY, O’Connell F, Flippin L, Günzler V, Horner JW, Depinho RA, Kaelin WG Jr (2006) Mouse model for noninvasive imaging of HIF prolyl hydroxylase activity: assessment of an oral agent that stimulates erythropoietin production. Proc Natl Acad Sci USA 103:105-110.

Sakami S, Maeda T, Bereta G, Okano K, Golczak M, Sumaroka A, Roman AJ, Cideciyan AV, Jacobson SG, Palczewski K (2011) Probing mechanisms of photoreceptor degeneration in a new mouse model of the common form of autosomal dominant retinitis pigmentosa due to $\mathrm{P} 23 \mathrm{H}$ opsin mutations. J Biol Chem 286: 10551-10567.

Stach L, Freemont PS (2017) The AAA+ ATPase p97, a cellular multitool. Biochem J 474:2953-2976.

van den Boom J, Meyer H (2018) VCP/p97-mediated unfolding as a principle in protein homeostasis and signaling. Mol Cell 69:182194.

Wójcik C, Rowicka M, Kudlicki A, Nowis D, McConnell E, Kujawa M, DeMartino GN (2006) Valosin-containing protein (p97) is a regulator of endoplasmic reticulum stress and of the degradation of $\mathrm{N}$-end rule and ubiquitin-fusion degradation pathway substrates in mammalian cells. Mol Biol Cell 17:4606-4618.

Yao J, Qiu Y, Frontera E, Jia L, Khan NW, Klionsky DJ, Ferguson TA, Thompson DA, Zacks DN (2018) Inhibiting autophagy reduces retinal degeneration caused by protein misfolding. Autophagy 14 $1226-1238$.

Ye Y, Meyer HH, Rapoport TA (2001) The AAA ATPase Cdc48/p97 and its partners transport proteins from the ER into the cytosol. Nature 414:652-656.

Zhang T, Mishra P, Hay BA, Chan D, Guo M (2017) Valosincontaining protein (VCP/p97) inhibitors relieve Mitofusindependent mitochondrial defects due to VCP disease mutants. Elife 6:e17834. 